\title{
A NOTE ON PREHARMONIC FUNCTIONS
}

A. C. ALLEN AND B. H. MURDOCH

1. Let $L$ be the set of points whose coordinates are rational integers. Let $D$ be a domain, that is to say, an open connected set, and let $G$ be the set $D \cdot L$. A point $P(m, n)$ of $G$ is an interior point if the four points $(m \pm 1, n),(m, n \pm 1)$ contiguous to $P$ belong to $G$. Otherwise $P$ is a boundary point.

A function $f(m, n)$ defined on $G$ is preharmonic if the value of $f$ at any interior point is the mean of the values of $f$ at the contiguous points, that is to say

$$
\begin{aligned}
4 f(m, n)= & f(m+1, n)+f(m-1, n)+f(m, n+1) \\
& +f(m, n-1) .
\end{aligned}
$$

For several decades the subject of preharmonic functions has been considered by many mathematicians, and the connection with harmonic functions has long been known. A recent paper by Heilbronn [1] states a number of theorems which are the analogues of classical theorems for harmonic functions.

In this note we consider functions which are preharmonic and nonnegative in the half-plane $n \geqq 0$ and prove a representation theorem analogous to that for positive harmonic functions [2], and a theorem which is the analogue of the Phragmén-Lindelöf type theorem for positive harmonic functions $[3 ; 4]$.

2. We require the following lemmas:

LEMмA 1. If $f(m, n)$ is preharmonic on a bounded domain $D$, then $f(m, n)$ is either constant or attains its maximum and minimum on $D$ on the boundary only.

Lemмa 2. If $f(m, n)$ is preharmonic everywhere and satisfies the inequality

$$
|f(m, n)|<A\left\{1+(|m|+|n|)^{k}\right\}
$$

for all $m, n$, where $k$ is a positive integer, then $f(m, n)$ is a polynomial of degree not exceeding $k$.

Received by the editors April 9, 1952 and, in revised form, April 13, 1953.

1 In what follows, $A$ will always represent a positive nonzero number, independent of the variables in the context. 
These lemmas are special cases of Theorems 1 and 6 of Heilbronn's paper.

LEMMA 3. The function

$$
h(m, n)=\frac{1}{\pi} \int_{0}^{\pi} \cos m t \phi^{n}(t) d t
$$

where $\phi(t)$ is the smaller root of the equation

$$
\phi(t)+\phi^{-1}(t)+2 \cos t=4,
$$

is preharmonic everywhere with the following properties:

(a) $h(0,0)=1$,

(b) $h(m, 0)=0$ for $m \neq 0$,

(c) $h(m, n)>0$ for $n>0$,

(d) $\left|h(m, n)-n / \pi\left(m^{2}+n^{2}\right)\right|<A / n\left(m^{2}+n^{2}\right)$ for all $m$ and positive $n$.

(a) and (b) follow by inspection. To prove (c), let

$$
M(n)=\underset{|m|<\infty}{\operatorname{glb}} h(m, n)
$$

for $n \geqq 0$. It is easily seen that $|\phi(t)|<1$ for $0<t \leqq \pi$ and so $M(n) \rightarrow 0$ as $n \rightarrow \infty$ and, from the difference equation for preharmonic functions, we have for $n \geqq 1$

$$
2 M(n) \geqq M(n+1)+M(n-1)
$$

and, since $M(0)=0$, the result follows.

It may be verified that $\phi(t)$ is a positive decreasing function of $t$ in $(0, \pi)$ with derivatives of all orders there, that

$$
\begin{gathered}
\phi^{\prime}(\pi)=0, \quad \lim _{t \rightarrow 0+} \phi^{\prime}(t)=-1, \\
\phi(t)=1-t+t^{2} / 2-t^{3} / 12+O\left(t^{4}\right)
\end{gathered}
$$

as $t \rightarrow 0+$, and that there exists a real number $\eta>0$ such that

$$
\phi(t) \leqq e^{-\eta t} \quad \text { for } 0 \leqq t \leqq \pi .
$$

Integrating by parts twice in the expression for $h(m, n)$ we have from (1) and the fact that $\sin m \pi=0$

$$
\pi h(m, n)=\frac{n}{m^{2}}-\frac{n}{m^{2}} \int_{0}^{\pi} \phi^{n-2}(t) \cos m t\left[(n-1)\left\{\phi^{\prime}(t)\right\}^{2}+\phi(t) \phi^{\prime \prime}(t)\right] d t,
$$

or, adding $\pi\left(n^{2} / m^{2}\right) h(m, n)$ to each side, 


$$
\pi \cdot \frac{m^{2}+n^{2}}{m^{2}} \cdot h(m, n)=\frac{n}{m^{2}}-\frac{n}{m^{2}} \int_{0}^{\pi} \phi^{n-2}(t) \psi(t) \cos m t d t,
$$

where

$$
\psi(t)=(n-1)\left\{\phi^{\prime}(t)\right\}^{3}+\phi(t) \phi^{\prime \prime}(t)-n \phi^{2}(t) .
$$

From the enunciated properties of $\phi(t)$ we may easily show that

$$
|\psi(t)|<A\left(|n| t^{2}+t\right)
$$

for $0<t \leqq \pi$. Thus, by (3), we have for $n \geqq 1$

$$
\begin{aligned}
\left|\pi \cdot \frac{m^{2}+n^{2}}{m^{2}} \cdot h(m, n)-\frac{n}{m^{2}}\right| & <A \frac{n}{m^{2}} \int_{0}^{\pi} e^{-n \eta t}\left(n t^{2}+t\right) d t \\
& <\frac{A}{n m^{2}}
\end{aligned}
$$

and this completes the proof of Lemma 3.

3. THEOREM 1. A necessary and sufficient condition for a function $f(m, n)$ to be non-negative and preharmonic for $n \geqq 0$ is that the numbers $f(m, 0)\{m=0, \pm 1, \pm 2, \cdots\}$ should be non-negative and satisfy

$$
\sum_{m=-\infty}^{\infty} \frac{f(m, 0)}{1+m^{2}}<\infty
$$

and that there should exist a non-negative number $D$ for which

$$
f(m, n)=D n+\sum_{r=-\infty}^{\infty} f(r, 0) h(m-r, n)
$$

for $n \geqq 0$.

Sufficiency. For any large positive $N$ and $n>0$ we have, from Lemma 3(d),

$$
\begin{aligned}
\sum_{r=-N}^{N} f(r, 0) h(m-r, n) & <A \sum_{r=-N}^{N} \frac{f(r, 0) n}{(m-r)^{2}+n^{2}} \\
& <A C(m, n) \sum_{r=-N}^{N} \frac{f(r, 0)}{1+r^{2}}
\end{aligned}
$$

where

$$
C(m, n)=\operatorname{lub}_{|r|<\infty} \frac{n\left(1+r^{2}\right)}{(m-r)^{2}+n^{2}}
$$

and is finite for any fixed $m, n$. Thus the function defined by (4) is 
an absolutely convergent series of non-negative preharmonic functions and, hence, is itself non-negative and preharmonic for $n \geqq 0$.

Necessity. Let $R$ be a positive integer and define

$$
f_{R}(m, n)=f(m, n)-\sum_{r=-R}^{R} f(r, 0) h(m-r, n) .
$$

Evidently $f_{R}(m, n)$ is preharmonic in the half-plane $n \geqq 0$ and also

$$
f_{R}(m, n) \geqq-\left\{\max _{|r| \leqq R} h(m-r, n)\right\} \sum_{r=-R}^{R} f(r, 0) .
$$

From Lemma 3(d) the right-hand side has arbitrarily small modulus for all points $(m, n)$ of the half-plane lying outside a sufficiently large circle with centre at $(0,0)$. Since, by Lemma 1 , a preharmonic function in a finite domain attains its minimum on the boundary and $f_{R}(m, n)=0$ for $n=0$, it follows that for $n \geqq 0, f_{R}(m, n) \geqq 0$. That is to say, for $n \geqq 0$ we have

$$
f(m, n) \geqq \sum_{r=-R}^{R} f(r, 0) h(m-r, n),
$$

and letting $R \rightarrow \infty$

$$
f(m, n) \geqq \sum_{r=-\infty}^{\infty} f(r, 0) h(m-r, n) .
$$

Next, by Lemma 3(d), there exists a large positive integer $N$ for which

$$
h(m, N)>1 /\left(N^{2}+m^{2}\right)
$$

for all integers $m$. Thus we have, from (5),

$$
\begin{aligned}
f(0, N) & \geqq \sum_{r=-\infty}^{\infty} f(r, 0) h(-r, N) \\
& \geqq \sum_{r=-\infty}^{\infty} \frac{f(r, 0)}{r^{2}+N^{2}} \\
& \geqq \frac{1}{N^{2}} \sum_{r=-\infty}^{\infty} \frac{f(r, 0)}{1+r^{2}} .
\end{aligned}
$$

This proves that if $f(m, n)$ is non-negative and preharmonic for $n \geqq 0$,

If we write

$$
\sum_{r=-\infty}^{\infty} \frac{f(r, 0)}{1+r^{2}}<\infty
$$




$$
f_{\infty}(m, n)=f(m, n)-\sum_{r=-\infty}^{\infty} f(r, 0) h(m-r, n),
$$

it remains to show that $f_{\infty}(m, n)=D n$ for some non-negative $D$. Now since the series $\sum_{r=-\infty}^{\infty} f(r, 0) h(m-r, n)$ is convergent and each term is non-negative and preharmonic for $n \geqq 0, f_{\infty}(m, n)$ also is non-negative and preharmonic for $n \geqq 0$, and, a fortiori, for any integral $t>0, f_{\infty}(m, n+t)$ is non-negative and preharmonic for $n \geqq 0$. From what we have just proved above, we have

$$
\sum_{r=-\infty}^{\infty} \frac{f_{\infty}(r, t)}{1+r^{2}}<\infty
$$

and, a fortiori, $f_{\infty}(m, t)<K_{t}\left(1+m^{2}\right)$, where $K_{t}$ is finite for each integral $t$. Let us assume for the moment that we have shown that

$$
f_{\infty}(m, n)<A n^{2}\left(1+m^{2}\right)<A\left[1+(|m|+|n|)^{4}\right]
$$

for $n>0$. We may continue $f_{\infty}(m, n)$ uniquely throughout the entire plane by writing

$$
f_{\infty}(m,-n)=-f_{\infty}(m, n)
$$

for $n>0$, and have

(i) $f_{\infty}(m, n)$ preharmonic everywhere,

(ii) $f_{\infty}(m, n)<A\left[1+(|m|+|n|)^{4}\right]$ everywhere,

(iii) $f_{\infty}(m, 0)=0$ for all $m$,

(iv) $\operatorname{sign} f_{\infty}(m, n)=\operatorname{sign} n$ for $n \neq 0$.

Applying Lemma 2 to $f_{\infty}(m, n)$ it follows from (8)(ii) that $f_{\infty}(m, n)$ is a polynomial of degree not exceeding 4 . From (8)(iii), $n$ must be a factor of $f_{\infty}(m, n)$; since $f_{\infty}(m, n)$ by $(7)$ contains only odd powers of $n$ we must have

$$
f_{\infty}(m, n)=n \phi\left(m, n^{2}\right),
$$

where $\phi\left(m, n^{2}\right)$ is a polynomial of degree not exceeding 3. Further, from (8)(iv), $\phi\left(m, n^{2}\right)$ is everywhere non-negative, and so of degree not exceeding 2 . We have now show'n that

$$
f_{\infty}(m, n)=n\left(\alpha m^{2}+\beta n^{2}+\gamma m+\delta\right)
$$

where $\alpha$ and $\beta$ are non-negative. It may be verified, from the difference equation, that since $f_{\infty}(m, n)$ is preharmonic, then $\alpha+3 \beta=0$. Thus $\alpha=\beta=0$ and this implies that $\gamma=0$ and $\delta \geqq 0$. This completes the proof that 


$$
f_{\infty}(m, n)=D n
$$

for some non-negative $D$.

It remains to prove (6). Consider the function

$$
g(m, n, \bar{m}, 2 \bar{n})=\frac{1}{\bar{n}} \sum_{r=1}^{2 \bar{n}} \sin \frac{r \pi(m-\bar{m}+\bar{n})}{2 \bar{n}} \cdot \sin \frac{r \pi}{2} \cdot \frac{\sinh \alpha_{r} n}{\sinh 2 \alpha_{r} \bar{n}}
$$

where $\alpha_{r}$ is the positive root of the equation

$$
\cosh \alpha_{r}+\cos \frac{r \pi}{2 \bar{n}}=2
$$

This function is preharmonic everywhere ${ }^{2}$ and may be shown to satisfy

$$
g(m, n, \bar{m}, 2 \bar{n})= \begin{cases}0 & \text { for } m=\bar{m} \pm \bar{n} \\ 0 & \text { for } n=0 \\ 0 & \text { for } 1 \leqq|m-\bar{m}| \leqq \bar{n}, n=2 \bar{n} \\ 1 & \text { for } m=\bar{m}, n=2 \bar{n} .\end{cases}
$$

Further,

From (9) we have

$$
\begin{aligned}
g(\bar{m}, 1, \bar{m}, 2 \bar{n}) & =\frac{1}{\bar{n}} \sum_{r=1}^{2 \bar{n}} \sin ^{2} \frac{r \pi}{2} \cdot \frac{\sinh \alpha_{r}}{\sinh 2 \alpha_{r} \bar{n}} \\
& \geqq \frac{1}{\bar{n}} \frac{\sinh \alpha_{1}}{\sinh 2 \alpha_{1} \bar{n}} .
\end{aligned}
$$

and so

$$
\cosh \alpha_{r}=2-\cos \frac{r \pi}{2 \pi}<\cosh \frac{r \pi}{2 \pi},
$$

$$
\alpha_{r}<\frac{r \pi}{2 \pi},
$$

and substituting this in the inequality for $g(\bar{m}, 1, \bar{m}, 2 \bar{n})$ we deduce that

$$
g(\bar{m}, 1, \bar{m}, 2 \bar{n})>A / \bar{n}^{2} .
$$

Let us suppose that (6) is not true. Then there exists an increasing sequence of integers $\left\{n_{\nu}\right\}$, and a corresponding sequence of integers $\left\{m_{\nu}\right\}$ such that as $\nu \rightarrow \infty$

2 This method of writing preharmonic functions as a sum of products is due to Phillips and Wiener [5]. 


$$
\frac{f_{\infty}\left(m_{\nu}, n_{v}\right)}{n_{\nu}^{2}\left(1+m_{\nu}^{2}\right)} \rightarrow \infty .
$$

We shall suppose first that the integers $n$, are even. Consider the function

$$
\bar{f}_{\nu}(m, n)=f_{\infty}(m, n)-f_{\infty}\left(m_{\nu}, n_{\nu}\right) g\left(m, n, m_{\nu}, n_{v}\right) .
$$

From (10) and (11) it is apparent that $\bar{f}_{\nu}(m, n) \geqq 0$ on the boundary of the square $\left|m-m_{\nu}\right| \leqq n_{\nu}, 0 \leqq n \leqq n_{\nu}$, and also, by Lemma 1 , inside the square. In particular, we have

$$
f_{\infty}\left(m_{\nu}, 1\right) \geqq f_{\infty}\left(m_{\nu}, n_{v}\right) g\left(m_{\nu}, 1, m_{\nu}, n_{v}\right),
$$

and so, by (11),

$$
f_{\infty}\left(m_{v}, n_{v}\right)<A n_{\nu}^{2}\left(1+m_{\nu}^{2}\right)
$$

which contradicts our assumption. Similarly, if the sequence is odd, we may show that

$$
f_{\infty}\left(m_{\nu}, n_{v}\right)<A n_{\nu}^{2}\left(1+m_{\nu}^{2}\right) \text {. }
$$

CoRollary. Suppose $f(m, n)$ to be preharmonic and non-negative in $n \geqq 0$. Then, as $n \rightarrow \infty$ subject to the condition $a m+b n=0$ where $a$ and $b$ are integers,

$$
f(m, n)-H(m, n)=D n+O\left\{\left(m^{2}+n^{2}\right)^{-1 / 2}\right\},
$$

where $D$ is a non-negative number and

$$
H(m, n)=\frac{1}{\pi} \sum_{r=-\infty}^{\infty} f(r, 0) \cdot \frac{n}{(m-r)^{2}+n^{2}} .
$$

This result follows immediately from Theorem 1 and Lemma 3(d).

4. If $H\left(r e^{i \theta}\right)$ is positive and harmonic in the half-plane $0<\theta<\pi$, then it may be written as [2]

$$
H\left(r e^{i \theta}\right)=d r \sin \theta+\frac{1}{\pi} \int_{-\infty}^{\infty} \frac{r \sin \theta}{r^{2}-2 r t \cos \theta+t^{2}} \cdot d g(t)
$$

where $d$ is a non-negative number and $g(t)$ is a nondecreasing function such that

$$
\int_{-\infty}^{\infty} \frac{d g(t)}{1+t^{2}}<\infty .
$$

LEMma 4. If $H\left(r e^{i \theta}\right)$ is defined by (12), $-1<\rho<1,0<\phi<\pi, n$ is an integer and $\alpha, \delta$ are any positive numbers such that 


$$
H\left(n \delta e^{i \phi}\right) \sim \alpha(n \delta)^{\rho}
$$

as $n \rightarrow \infty$, then

$$
H\left(r e^{i \phi}\right) \sim \alpha r^{p}
$$

as $r \rightarrow \infty$.

There is no loss in generality in assuming $d=0$, and so as $n \rightarrow \infty$

$$
\frac{1}{\pi} \int_{-\infty}^{\infty} \frac{d g(t)}{(n \delta)^{2}-2 n \delta t \cos \phi+t^{2}} \sim \alpha \operatorname{cosec} \phi \cdot(n \delta)^{\rho-1}
$$

This is easily shown to imply that for $x>1$

$$
\frac{g(x)-g(-x)}{x^{2}}+\int_{x}^{\infty} \frac{d\{g(t)-g(-t)\}}{x^{2}+t^{2}}<A x^{0-1} .
$$

Further, it will be sufficient to prove that for $|r-\sigma| \leqq 1$ and $r \rightarrow \infty$

$$
\Psi\left(r e^{i \phi}\right)-H\left(\sigma e^{i \phi}\right)=o\left(r^{p}\right) .
$$

Now from (12), since $d=0$,

$$
\begin{aligned}
& \left|H\left(r e^{i \phi}\right)-H\left(\sigma e^{i \phi}\right)\right| \\
& =\left|\frac{(r-\sigma) \sin \phi}{\pi} \int_{-\infty}^{\infty} \frac{\left(t^{2}-r \sigma\right) d g(t)}{\left(r^{2}-2 r t \cos \phi+t^{2}\right)\left(\sigma^{2}-2 \sigma t \cos \phi+t^{2}\right)}\right| \\
& \leqq A\left[\frac{g(r)-g(-r)}{r^{2}}+\int_{r}^{\infty} \frac{d\{g(t)-g(-t)\}}{r^{2}+t^{2}}\right] \\
& \leqq A r^{\rho-1} \\
& =o\left(r^{p}\right)
\end{aligned}
$$

The following two lemmas are contained in a paper by Allen and Kerr $[4]:{ }^{8}$

Lemma 5. If $H\left(r e^{i \theta}\right)$ is defined by (12), $-1<\rho<1$, and

$$
H\left(r e^{i \pi / 2}\right) \sim(1+\rho) \alpha r^{\rho} \sec \rho \pi / 2
$$

as $r \rightarrow \infty$, then

$$
g(x)-, g(-x) \sim 2 \alpha x^{1+p}
$$

as $x \rightarrow \infty$.

3 Actually Allen and Kerr state their results for the case $r \rightarrow 0+$, but the case $r \rightarrow \infty$ is an elementary corollary. 
LEMMA 6. If $H\left(r e^{i \theta}\right)$ is defined by (12), $-1<\rho<1$, and

$$
H\left(r e^{i \theta}\right) \sim(1+\rho) \operatorname{cosec} \rho \pi[\alpha \sin \rho(\pi-\theta)+\beta \sin \rho \theta] r^{\rho},
$$

as $r \rightarrow \infty$ for two distinct values of $\theta$, then (13) remains true for all values of $\theta$ and as $x \rightarrow \infty$

$$
g(x)-g(0) \sim \alpha x^{1+\rho}, \quad g(0)-g(-x) \sim \beta x^{1+p} .
$$

THEOREM 2. If $f(m, n)$ is non-negative and preharmonic in the halfplane $n \geqq 0,-1<\rho<1$, and

$$
f(0, n) \sim(1+\rho) \alpha \sec \rho \pi / 2 \cdot n^{\rho}
$$

as $n \rightarrow \infty$, then

$$
\sum_{m=-R}^{R} f(m, 0) \sim 2 \alpha R^{1+\rho}
$$

as $R \rightarrow \infty$.

TheoRem 3. If $f(m, n)$ is non-negative and preharmonic in the halfplane $n \geqq 0,-1<\rho<1$, and

$$
\begin{aligned}
f(m, n) \sim(1+\rho) \operatorname{cosec} \rho \pi & {\left[\alpha \sin \rho\left(\pi-\arctan \frac{n}{m}\right)\right.} \\
& \left.+\beta \sin \rho\left(\arctan \frac{n}{m}\right)\right]\left(n^{2}+m^{2}\right)^{\rho / 2}
\end{aligned}
$$

as $n \rightarrow \infty$ for two distinct rational values of $n / m$, then (14) remains true for all rational values of $n / m$, and as $R \rightarrow \infty$ we have

$$
\sum_{m=0}^{R} f(m, 0) \sim \alpha R^{1+\rho}, \quad \sum_{m=-R}^{0} f(m, 0) \sim \beta R^{1+\rho} .
$$

In (12) we define $g(x)$ to be constant in the interval $n<x<n+1$, for all integers $n$ and with saltus $f(n, 0)$ at $x=n$ : then Theorems 2 and 3 follow directly from the corollary to Theorem 1 and Lemmas 4,5 , and 6.

THEOREM 4. If $f(m, n)$ is non-negative and preharmonic in the halfplane $n \geqq 0$, and for some finite $\bar{n} \geqq 0$

$$
\lim _{m \rightarrow \infty} f(m, \bar{n})=\alpha,
$$

then we have

$$
\lim _{m \rightarrow \infty} f(m, n)=\alpha_{n}
$$


for $n \geqq 0$ where $\alpha_{n}$ is a linear function of $n$.

$f(m, n)$ is non-negative and preharmonic in the half-plane $n \geqq \bar{n}$ and so by Theorem 1 has the representation

$$
f(m, \bar{n}+p)=D_{p}+\sum_{r=-\infty}^{\infty} f(r, \bar{n}) h(m-r, p)
$$

for $p>0$, and some non-negative $D$. From the definition of $h(m, n)$ it is easily verified that

$$
\sum_{m=-\infty}^{\infty} h(m, p)=1
$$

Also, from Lemma 3(d), for $p>0$

$$
h(m, p)<A p /\left(m^{2}+p^{2}\right) \text {. }
$$

From the hypothesis and Theorem 1, given $\epsilon>0$ there exists an integer $N>0$ for which

$$
|f(m, \bar{n})-\alpha| \leqq \epsilon
$$

for $m>N$ and for which

$$
\sum_{r=-\infty}^{-N} \frac{f(r, \bar{n})}{1+r^{2}} \leqq \epsilon .
$$

We may now apply (15)-(18) as follows:

$$
\begin{aligned}
|f(m, \bar{n}+p)-D p-\alpha| & \leqq \sum_{r=-\infty}^{\infty}|f(r, \bar{n})-\alpha| h(m-r, p) \\
\leqq & \sum_{r=-\infty}^{N} f(r, \bar{n}) h(m-r, p)+\alpha \sum_{r=-\infty}^{N} h(m-r, p) \\
& +\sum_{r=-N+1}^{\infty}|f(r, \bar{n})-\alpha| h(m-r, p) \\
\leqq & A p \sum_{r=-\infty}^{-N} \frac{f(r, \tilde{n})}{1+r^{2}}+\frac{A p}{(m-N)^{2}} \sum_{r=-N}^{N} f(r, \tilde{n}) \\
& +A p \alpha \sum_{r=m-N}^{\infty} \frac{1}{r^{2}+p^{2}}+\epsilon \sum_{r=N+1}^{\infty} h(m-r, p) \\
\leqq & A p \epsilon+\frac{A p}{(m-N)^{2}} \sum_{r=-N}^{N} f(r, \tilde{n})+\frac{A p \alpha}{m-N}+\epsilon .
\end{aligned}
$$


It is apparent that by a suitable choice of $\epsilon$ and correspondingly large $m$, the right-hand side is arbitrarily small. This proves the theorem for $n>\bar{n}$ and the complete result follows from the difference equation for preharmonic functions.

We are grateful to a referee for helpful criticism of the presentation of this paper. One of us (A.C.A) is indebted to the Commonwealth Fund of New York for a Commonwealth Fellowship.

\section{REFERENCES}

1. H. A. Heilbronn, Proc. Cambridge Philos. Soc. vol. 45 (1949) pp. 194-206.

2. L. H. Loomis and D. V. Widder, Duke Math. J. vol. 9 (1942) pp. 643-645.

3. L. H. Loomis, Trans. Amer. Math. Soc. vol. 53 (1943) pp. 239-250.

4. A. C. Allen and E. Kerr, J. London Math. Soc. vol. 28 (1953) pp. 80-89.

5. H. B. Phillips and N. Wiener, Journal of Mathematics and Physics vol. 2 (1923) pp. 105-124.

Princeton University

\section{A THEOREM OF PHRAGMEN-LINDELÖF TYPE ${ }^{1}$}

\section{ALFRED HUBER}

1. Introduction. In the present paper the Phragmén-Lindelöf theorem for harmonic functions in the formulation of M. Heins [4] shall be extended to the solutions of the elliptic partial differential equation

$$
L_{k}[u] \equiv \sum_{1}^{n} \frac{\partial^{2} u}{\partial x_{i}^{2}}+\frac{k}{x_{n}} \frac{\partial u}{\partial x_{n}}=0 \quad(k<1)
$$

( $k$ denoting a real constant). Equation (1.1) appears in several problems. For an exposition of previous results in the theory of the solutions of (1.1) we refer to a recent paper of A. Weinstein [9].

A theorem of Phragmén-Lindelöf type for the solutions of a rather general class of elliptic partial differential equations has been proved by D. Gilbarg [3] and E. Hopf [5]. Because of the singular coefficient, (1.1) is not contained in this class.

We introduce the following notations, $P\left(x_{1}, x_{2}, \cdots, x_{n}\right)$ denoting a point in the $n$-dimensional space:

Presented to the Society, February 28, 1953; received by the editors March 30 , 1953.

1 Sponsored by the Office of Naval Research under contract Nr. N7 ONR-39705. 